\title{
MULTICULTURAL FAMILY HELP KIT
}

\author{
Suzanne Pope, Kym Scanlon and Beverley Raphael \\ Centre for Mental Health \\ NSW Department of Health
}

\section{Jan Heslep, Maria Cassaniti and John Spiteri \\ Transcultural Mental Health Centre, Parramatta}

The burden of mental health problems is large and increasing. Eighteen per cent of people aged over 18 years in Australia report experiencing a mental health problem or disorder (anxiety, affective or substance use disorders) in the past 12 months. Prevalence is highest for young people aged 18 to 25 years (27 per cent). Despite this, only a small percentage of people with mental disorders actually seek help (38 per cent). ${ }^{1}$ Further, there is uncertainty about the number of people requiring information about mental health issues. This highlights the importance of improving mental health literacy among the NSW population and establishing initiatives to promote mental health and prevent the onset of mental health problems and disorders. ${ }^{2-4}$ This article describes a new resource, the Multicultural Family Help Kit, that will help to improve the level of mental health literacy in NSW residents who are from non-English speaking backgrounds.

New South Wales has the most culturally diverse population in Australia. The State is home to 40 per cent of Australia's non-English speaking background (NESB) overseas-born population. ${ }^{5}$ In 1996, 27 per cent of the NSW population were born overseas, and were from a NESB. This equals a total of $1,015,862$ people aged five years and over who reported speaking a language other than English at home. ${ }^{5}$ Children, adolescents and their families from culturally and linguistically diverse backgrounds may be at risk for poor mental health outcomes due to a wide range of factors. These include:

- migration or refugee experiences

- intergenerational conflict

- grief and loss relating to separation from extended family

- post traumatic stress from experiences prior to resettlement; and possible experiences of racism and discrimination after resettlement. ${ }^{6}$

Further, recent research highlights that communities of NESB have a lower utilisation of mental health services. While these communities may be less inclined to use mental health services, the prevalence of mental health problems and disorders may not be less. ${ }^{7}$

The Multicultural Family Help Kit has been developed to assist people from culturally and linguistically diverse backgrounds to recognise the signs of mental health problems in children and young people and to seek help early. The kit, launched by the Minister in December 1999, will ensure families are better informed about mental health issues and the services that exist to assist them and their children. The kit is a collaborative initiative of the Transcultural Mental Health Centre (TMHC), NSW Department of Health and SBS Radio.

The TMHC provides a statewide service for people from culturally and linguistically diverse communities. Their role includes: the provision of clinical services; consultation to metropolitan and rural Area Health Services for developing appropriate multilingual mental health services; and, the development of multilingual mental health information and resources.

Bi-lingual community workers, multicultural community welfare agencies, ethnic community organisations and key community members, including young people, were consulted to ensure that the information and issues covered in the kit were sensitive to the cultures, values and practices for several different communities and languages.

The Multicultural Family Help Kit provides information on the following important topics:

- child and adolescent mental health problems

- prevention of suicide

- challenging behaviours

- psychosis

- body image and eating disorders

- post traumatic stress

- depression

- fears and anxiety.

The kit is available in a range of formats (audio-tape, print format and on the Web site) and was also developed as an SBS radio series. The SBS radio series was aired in November and December 1999 in 16 languages, including English, Arabic, Cantonese, Croatian, Filipino (Tagalog), Greek, Hindi, Italian, Korean, Macedonian, Mandarin, Serbian, Spanish, Tongan, Turkish and Vietnamese.

The radio program addressed mental health issues of concern for children and young people from non-English speaking backgrounds and their families, including: commonly held misunderstandings and myths about mental disorders, information on mental health services, and details of where to obtain the kit. A call for action was broadcast after each session giving listeners the opportunity to contact the Transcultural Mental Health Centre for services or further printed information.

The kit is being distributed to mental health professionals, community health centres, general practitioners, 
multicultural community welfare agencies, ethnic community organisations, early childhood centres, child and adolescent mental health services and other relevant services. The use of the kit by service providers and feedback from consumers on the relevance and usefulness of the information will be monitored and evaluated by the TMHC. This information will be used to inform best practice in the area of cross-cultural health information provision.

The Multicultural Family Help Kit is an important new resource that will contribute towards the mental health information needs of culturally and linguistically diverse communities in NSW.

\section{REFERENCES}

1. Andrews G, Hall W, Teesson M, Henderson S. National Survey of Mental Health and Wellbeing, Report 2: The Mental Health of Australians. Canberra: Mental Health Branch, Commonwealth Department of Health and Aged Care, 1999.
2. For a definition of mental health literacy refer to Jorm AF, Korten AE, Jacomb PA, Christensen H, Rogers B, Pollit P. Mental health literacy: A survey of the public's ability to recognise mental disorders and their beliefs about the effectiveness of treatment. Med J Aust, 1997;166:182-186.

3. Haggerty Mrazek PJ, Haggerty RJ. Reducing risks for mental disorders: Frontiers for preventive intervention research. Washington DC: National Academy Press, 1994.

4. Marshall J. and Watt P. Child behaviour problems: A literature review of its size and nature and prevention interventions. Perth: Interagency Committee on Children's Futures, 1999.

5. Australian Bureau of Statistics. Census Data, 1996. ABS Catalogue No. 2015.0.

6. Mihalopoulos C, Pirkis J. Investigation and further development of the role of general practitioners and other primary care agencies in the delivery of mental health services to NESB consumers and their families. Melbourne: Australian Transcultural Mental Health Network, 1998.

7. McDonald R, Steel Z. Immigrants and mental health: An epidemiological analysis. Sydney: Transcultural Mental Health Centre, 1997.

The various formats of the Multicultural Family Help Kit can be ordered as follows:

Copies of the eight print versions of the kit in Arabic, Chinese (both Cantonese and Mandarin), Greek, Italian, Korean, Spanish, Turkish and Vietnamese can be ordered from: the Better Health Centre, PO Box 58, Gladesville, NSW 2111; Telephone: (02) 9816 0452; Facsimile: (02) 98160492.

The 16 audio tapes are available from: the Transcultural Mental Health Centre, LMB 7118, Parramatta BC, NSW 2150; Telephone: (02) 9840 3800; Facsimile: (02) 98403755.

From April 2000 the other six language versions of the kit (Serbian, Macedonian, Hindi, Croatian, Filipino and Tongan) can be downloaded from the TMHC Web site at www.tmhc.nsw.gov.au, or from the Multicultural Health Communication Service Web site at www.mhcs.health.nsw.gov.au.

\section{REFUGEE HEALTH IN NSW}

\section{Mitchell Smith}

\section{Director, NSW Refugee Health Service}

Each year over 4,000 humanitarian entrants settle in NSW. They have endured persecution and violations of their rights as human beings, sometimes torture, and almost always psychological trauma. Most have had significantly limited access to medical and dental care. Some have been exposed to adverse environmental conditions while fleeing or in refugee camps. In terms of health, refugees and other migrants with refugee-like experiences are recognised as being one of the most vulnerable groups in our society.

Source countries have altered over the decades, from the post-war and 1960s European migrations, through the South East Asian phases of the late 1970s and 1980s, to the more recent intakes from Iraq, Iran, the former Yugoslavia, and the Horn of Africa. Two-thirds of humanitarian migrants to NSW settle in the Greater West of Sydney. Smaller numbers live across metropolitan Sydney, in the Hunter and Illawarra Areas, and throughout rural areas in towns as diverse as Bathurst, Coffs Harbour and Wagga Wagga.

The NSW Department of Health has recently produced a blueprint, the first of its kind in Australia, which outlines an integrated and coordinated approach to the health needs of refugees. Called Strategic Directions in Refugee Health Care in $N S W,{ }^{1}$ the document provides a framework to guide existing service provision, and to promote collaboration in responding to the diverse health service needs of refugees. 